\title{
Observation of Weyl nodes in TaAs
}

\author{
B. Q. Lv ${ }^{1,2 \dagger}$, N. Xu ${ }^{2,3 \dagger}$, H. M. Weng ${ }^{1,4}$, J. Z. Ma ${ }^{1,2}$, P. Richard ${ }^{1,4}$, X. C. Huang', L. X. Zhao', G. F. Chen ${ }^{1,4}$, \\ C. E. Matt ${ }^{2}$, F. Bisti ${ }^{2}$, V. N. Strocov ${ }^{2}$, J. Mesot ${ }^{2,3,5}$, Z. Fang ${ }^{1,4}$, X. Dai ${ }^{1,4}$, T. Qian ${ }^{1 \star}$, M. Shi $^{2 \star}$ and H. Ding ${ }^{1,4 \star}$
}

\begin{abstract}
In 1929, H. Weyl proposed that the massless solution of the Dirac equation represents a pair of a new type of particles, the so-called Weyl fermions ${ }^{1}$. However, their existence in particle physics remains elusive after more than eight decades. Recently, significant advances in both topological insulators and topological semimetals have provided an alternative way to realize Weyl fermions in condensed matter, as an emergent phenomenon: when two non-degenerate bands in the three-dimensional momentum space cross in the vicinity of the Fermi energy (called Weyl nodes), the low-energy excitations behave exactly as Weyl fermions. Here we report the direct observation in TaAs of the long-sought-after Weyl nodes by performing bulk-sensitive soft $X$-ray angle-resolved photoemission spectroscopy measurements. The projected locations at the nodes on the (001) surface match well to the Fermi arcs, providing undisputable experimental evidence for the existence of Weyl fermionic quasiparticles in TaAs.
\end{abstract}

The massless Dirac equation in the three-dimensional (3D) momentum space can be regarded as the overlap of two Weyl fermions with opposite chirality ${ }^{1,2}$. The Dirac fermionic quasiparticle is stable under the protection of some crystal symmetry in topological Dirac semimetals such as $\mathrm{Na}_{3} \mathrm{Bi}$ (ref. 3) and $\mathrm{Cd}_{3} \mathrm{As}_{2}$ (ref. 4). However, a separated single Weyl node is much more robust and requires no protection of crystal symmetry. An isolated Weyl node is a sink or source of gauge field of Berry curvature, like a monopole in momentum space, and the chirality corresponds to its topological charge $\mathrm{e}^{5-7}$. Weyl nodes appear in pairs of opposite chirality in a real material due to the 'No-go theorem ${ }^{8,9}$. To obtain isolated Weyl nodes, the spin degeneracy of the electronic bands has to be removed by breaking either inversion symmetry or time-reversal symmetry. Although non-degenerate band crossing is not rare, finding a material with only Weyl nodes near the Fermi energy $\left(E_{\mathrm{F}}\right)$ is a big challenge. Recently, the noncentrosymmetric and non-magnetic transition-metal monoarsenide TaAs has been predicted to be a Weyl semimetal (WSM), and twelve pairs of Weyl nodes are expected in its 3D Brillouin zone (BZ; refs 10,11). Compared with other proposals ${ }^{6,7,12-22}$ for realizing a Weyl state, the TaAs family features easy sample fabrication, a non-magnetic state and no fine-tuning of the electronic states is necessary, making experimental studies of Weyl semimetals possible. Many exotic properties induced by the Weyl nodes have been predicted and observed recently, such as surface states with Fermi $\operatorname{arcs}^{23,24}$ and a negative magneto-resistivity ${ }^{25,26}$ due to the chiral anomaly ${ }^{27-29}$. However, crucial evidence for Weyl nodes in the bulk states has not been observed. In this paper, by using soft X-ray angle-resolved photoemission spectroscopy (ARPES), which is sensitive to the bulk states, we report the first experimental observation of Weyl nodes in TaAs.
TaAs crystallizes in a body-centred-tetragonal structure with the nonsymmorphic space group $I 4_{1} m d$. The lattice parameters are $a=b=3.4348 \AA$ and $c=11.641 \AA$ at $300 \mathrm{~K}$. The crystal structure consists of alternating Ta and As layers (Fig. 1a). The adjacent TaAs layers are rotated by $90^{\circ}$ and shifted by $a / 2$, leading to a lack of inversion symmetry. First-principles band calculations predict that TaAs is a time-reversal invariant 3D WSM with twelve pairs of Weyl nodes in the 3D BZ (Fig. 1c) ${ }^{10,11}$. These Weyl nodes with opposite chirality are linked to the Fermi arcs of the surface state in the projected surface BZ.

Fermi-arc-like surface states have been identified in recent vacuum ultraviolet (VUV) ARPES experiments ${ }^{23,24}$, which are remarkably well reproduced by the band calculations for the As termination $^{23}$. However, due to the short escape depth of the photoelectrons excited by VUV light, the bulk electronic structure was nearly invisible in the VUV ARPES experiments. To probe the bulk electronic states of TaAs, we use soft X-rays to promote valence photoelectrons to a higher kinetic energy with a much longer escape depth, thus representing the bulk band structure. This bulk nature is reflected in the shallow core-level spectra shown in Fig. 2a, where the two As $3 d$ surface peaks, which are strong at photon energy $h \nu=70 \mathrm{eV}$, almost vanish at $h \nu=440 \mathrm{eV}$. In addition, due to the Heisenberg uncertainty principle, increasing the photoelectron escape depth sharpens the intrinsic experimental resolution in the surface-perpendicular momentum $\left(k_{z}\right.$; ref. 30$)$, allowing accurate navigation in the $3 \mathrm{D}$ momentum space.

The bulk nature of the electronic states is further confirmed by the strong $k_{z}$ dispersion on varying the incident photon energy (Fig. 2). The Fermi surfaces and band dispersions exhibit a modulation along the $k_{z}$ direction with a period of $2 \pi / c^{\prime}$, where $c^{\prime}$ is one half of the $c$-axis lattice constant of TaAs. The experimental bands along the $\Gamma-\mathrm{Z}$ and $\Gamma-\Sigma(\mathrm{S})-\mathrm{Z}$ high-symmetry lines are in remarkably good agreement with the bulk bands from firstprinciples calculations with spin-orbit coupling (SOC) included, as demonstrated in Fig. 2. Just below $E_{\mathrm{F}}$, there are two bands clearly observed along $\Sigma(\mathrm{S})-\Gamma$ in Fig. 2e, which are mostly composed of Ta $5 d$ orbitals and well reproduced by calculations. The large band splitting of these two bands is due to the Rashba-like SOC originating from broken inversion symmetry and intrinsic SOC in Ta $5 d$ electrons. The calculations without SOC show that the bands crossing near $E_{\mathrm{F}}$ within the mirror-invariant planes are protected by the mirror symmetry, leading to gapless nodal rings in the mirror plane $^{10}$. As the SOC is turned on, the nodal rings are fully gapped, generating non-zero mirror Chern numbers for the occupied states on the mirror planes and Weyl nodes slightly off the mirror planes ${ }^{10}$.

To identify the Weyl nodes and their locations in the momentum space, we mapped out the 3D electronic structure with a number of incident photon energies. The Fermi surface map at $h v=440 \mathrm{eV}$

${ }^{1}$ Beijing National Laboratory for Condensed Matter Physics and Institute of Physics, Chinese Academy of Sciences, Beijing 100190, China. ${ }^{2}$ Paul Scherrer Institute, Swiss Light Source, CH-5232 Villigen PSI, Switzerland. ${ }^{3}$ Institute of Condensed Matter Physics, École Polytechnique Fédérale de Lausanne, CH-1015 Lausanne, Switzerland. ${ }^{4}$ Collaborative Innovation Center of Quantum Matter, Beijing, China. ${ }^{5}$ Laboratory for Solid State Physics, ETH Zürich, CH-8093 Zürich, Switzerland. TThese authors contributed equally to this work. ^e-mail: tqian@iphy.ac.cn; ming.shi@psi.ch; dingh@iphy.ac.cn 
a

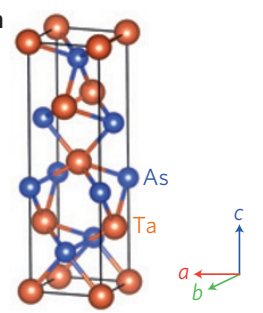

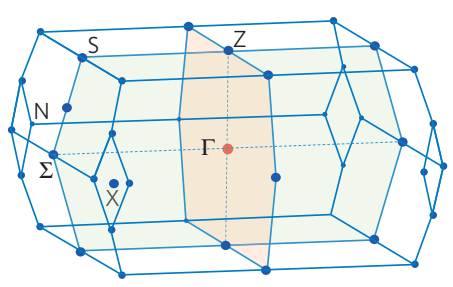

c

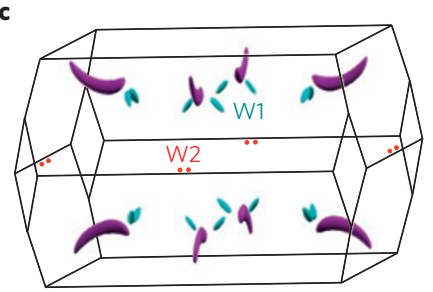

d

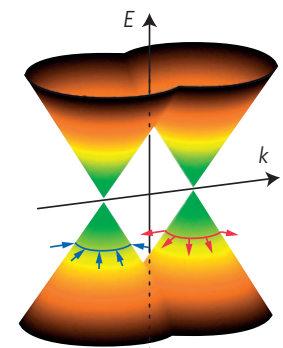

Figure 1 | Crystal structure and electronic structure of TaAs. a, Crystal structure of TaAs. b, Bulk BZ of TaAs with the high-symmetry points indicated. The shaded planes represent the mirror-invariant (100) and (010) planes. c, Fermi surfaces from first-principles calculations. The purple banana-like hole pockets are topologically trivial Fermi surfaces that do not enclose a Weyl node. The blue date-like electron pockets enclose the Weyl nodes W1, which are $21 \mathrm{meV}$ below $E_{\mathrm{F}}$. The Weyl nodes $\mathrm{W} 2$ in the $k_{\mathrm{z}}=0$ plane are only $2 \mathrm{meV}$ above $E_{\mathrm{F}}$ (the Fermi surfaces enclosing them are tiny and marked by the red dots). d, Schematic band dispersions of one pair of Weyl nodes in a 2D plane of the momentum space. The arrows indicate that the Weyl node is a monopole of Berry flux in the momentum space.

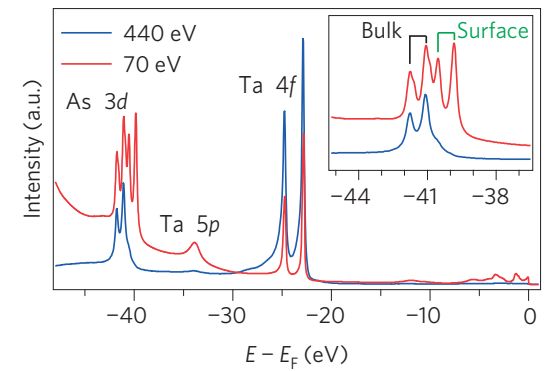

b

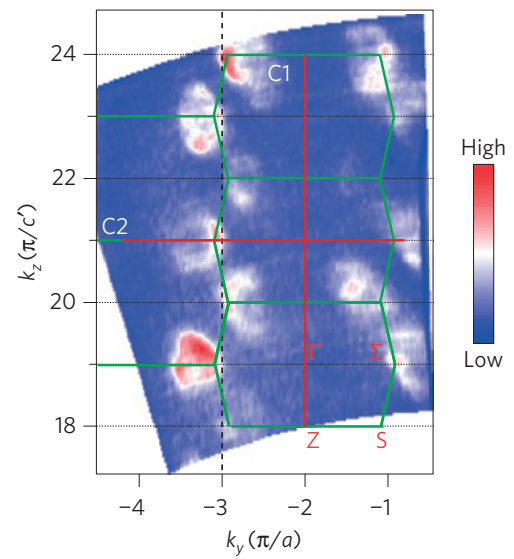

c
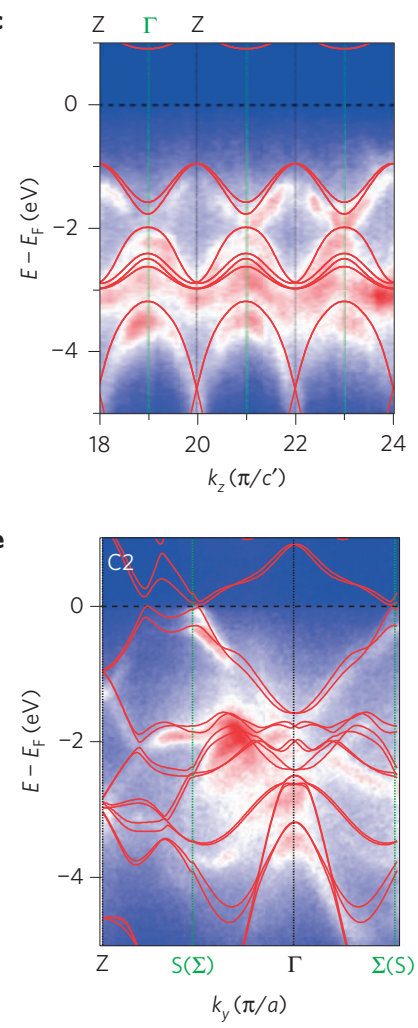

d

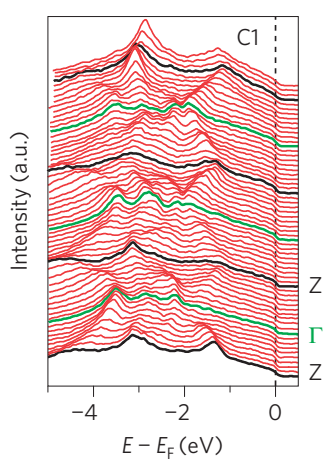

f

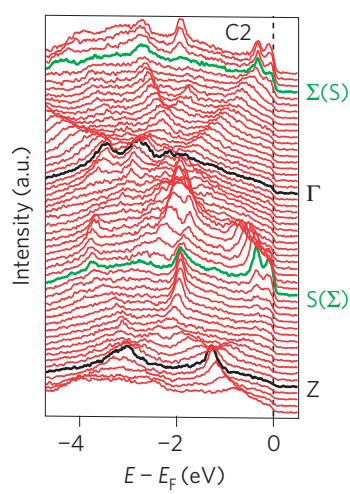

Figure 2 | Core-level spectra and electronic structure in the $\boldsymbol{k}_{\mathbf{x}}=0$ plane. a, Core-level spectra taken at $h v=70$ and $440 \mathrm{eV}$, respectively. The inset shows the magnification of the As $3 d$ core-level peaks, indicating that the surface electronic states are drastically suppressed with the incident soft $X$-rays. The peaks marked as 'Surface' are contributed by photoelectrons from the topmost As layer. b. Photoemission intensity plot at $E_{\mathrm{F}}$ in the $k_{y}-k_{z}$ plane at $k_{x}=0$. The green lines represent the BZ structure in the $k_{y}-k_{z}$ plane. $\mathbf{c}, \mathbf{d}$, Photoemission intensity plot (c) and energy distribution curves (d) along $\Gamma-Z$ (C1) indicated in $\mathbf{b}$. e,f, Photoemission intensity plot (e) and energy distribution curves (f) along $\Gamma-\Sigma(S)-Z(C 2)$ indicated in $\mathbf{b}$. For comparison, the calculated bands along $\Gamma-Z$ and $\Gamma-\Sigma(S)-Z$ are plotted on top of the experimental data in $\mathbf{c}$ and $\mathbf{e}$, respectively.

in Fig. 3b clearly shows two date-like pockets slightly off the mirror plane. The pockets originate from an ' $\mathrm{M}$ '-shaped band perpendicular to the (010) mirror plane (Fig. 3d,e), whose tops touch $E_{\mathrm{F}}$ at $k_{x}= \pm 0.06 \pi / a$. On moving along the $k_{y}$ direction, the two tops gradually merge together and sink below $E_{\mathrm{F}}$, with this band eventually evolving into a ' $\Lambda$ ' shape, as illustrated in Fig. $3 \mathrm{~h}$. The band parallel to the mirror plane through one of the pockets exhibits a ' $\Lambda$ ' shape with its top at $k_{y}=-3.53 \pi / a$ (Fig. 3f,g). These observed band dispersions in the $k_{x}-k_{y}$ plane are highly consistent with the calculated Weyl-cone band structure of W1.

To verify the $3 \mathrm{D}$ nature of the cone, we also investigate the band dispersion along the $k_{z}$ direction. In this case, as shown in Fig. 3i, the ' $M$ '-shaped band also evolves into a ' $\Lambda$ ' shape. These results demonstrate that these pockets enclose the $3 \mathrm{D}$ Weyl nodes $\mathrm{W} 1$ that are located at $( \pm 0.06, \pm 0.47, \pm 0.58)$ in units of $\left(\pi / a, \pi / a, \pi / c^{\prime}\right)$, which are pretty close to the calculated locations $( \pm 0.059, \pm 0.556, \pm 0.592)$ (ref. 10).

The predicted Weyl nodes near the BZ boundary (labelled as W2) are also identified in Fig. 4. As compared with the W1 nodes, the nodes of the W2 pair are very close to each other in the calculations ${ }^{10}$. This is consistent with our experimental results, in which we observe a nearly ' $\Lambda$ '-shaped band rather than an ' $M$ '-shaped band along the cut perpendicular to the mirror plane (Fig. 4c,d). The band also exhibits a ' $\Lambda$ ' shape along both the $k_{y}$ 


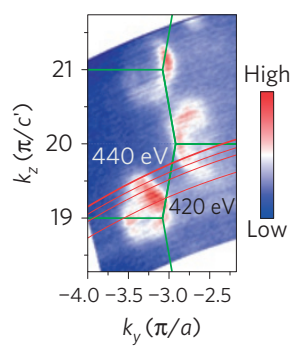

b

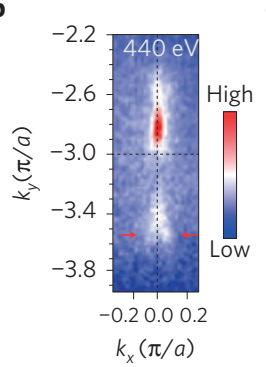

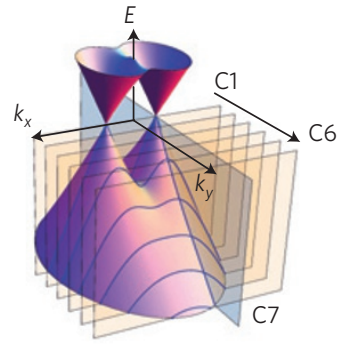

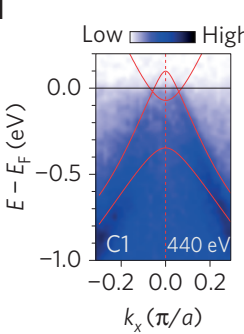

e Low

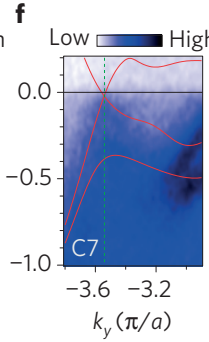

g

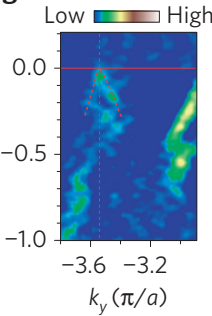

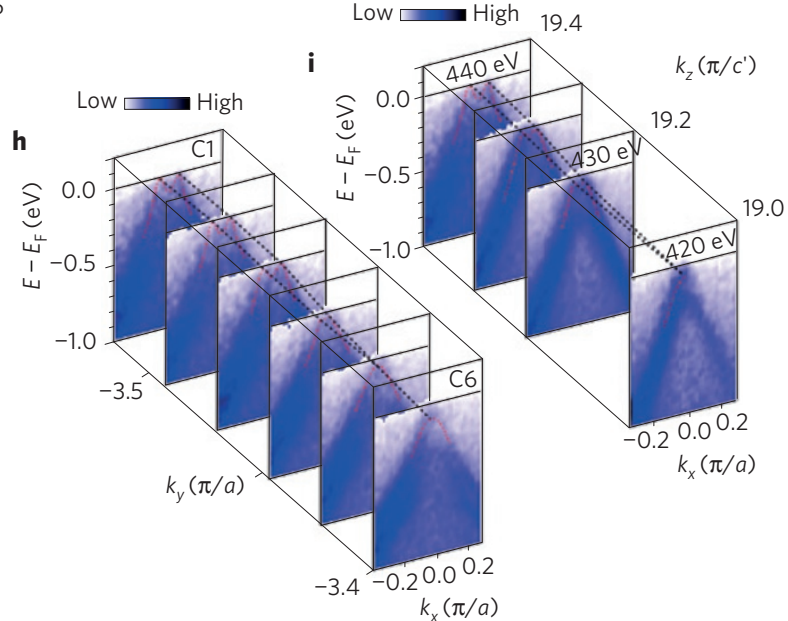

Figure 3 | Band dispersions near the Weyl nodes W1. a, Photoemission intensity plot at $E_{\mathrm{F}}$ in the $k_{y}-k_{z}$ plane at $k_{x}=0$. The four red curves from top to

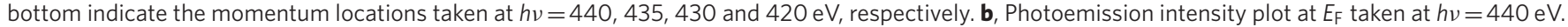
The red arrows indicate the date-like pockets that enclose the Weyl nodes W1. c, Schematic band dispersion of one pair of Weyl nodes in the $k_{x}-k_{y}$ plane. The vertical planes C1-C6 schematize the ARPES measurements that slice through the Weyl cone at different $k_{y}$ positions. d,e, Photoemission (d) and curvature intensity (e) plots along the $k_{x}$ direction through the two Weyl nodes W1, illustrating the 'M'-shape dispersion of a pair of Weyl nodes. $\mathbf{f}, \mathbf{g}$, Photoemission (f) and curvature intensity ( $\mathbf{g}$ ) plots along the $k_{y}$ direction through one of the Weyl nodes W1. For comparison, the calculated bands through the Weyl nodes W1 along the $k_{x}$ and $k_{y}$ directions are plotted (red curves) on top of the experimental data in $\mathbf{d}$ and $\mathbf{f}$, respectively. Note that the calculated bands in $\mathbf{d}$ and $\mathbf{f}$ are shifted by $0.11 \pi / a$ along the $k_{y}$ direction to be consistent with the experimental bands. $\mathbf{h}$, Band dispersions along the $k_{x}$ direction at different $k_{y}$ positions. i, Band dispersions along the $k_{x}$ direction at different $k_{z}$ positions. The dotted lines in $\mathbf{h}$ and $\mathbf{i}$, which are guides to the eye indicating the band dispersions, show the evolution of the band shape. The dashed lines in $\mathbf{h}$ and $\mathbf{i}$ track the band tops.

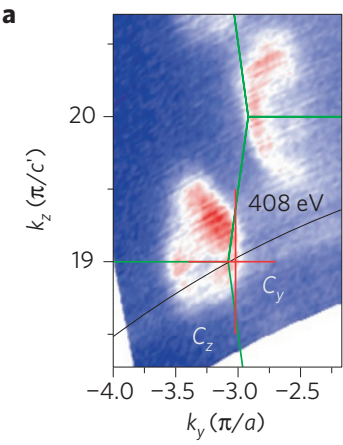

b

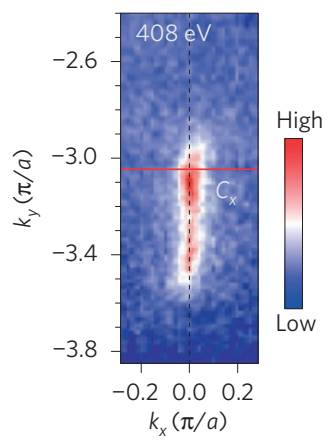

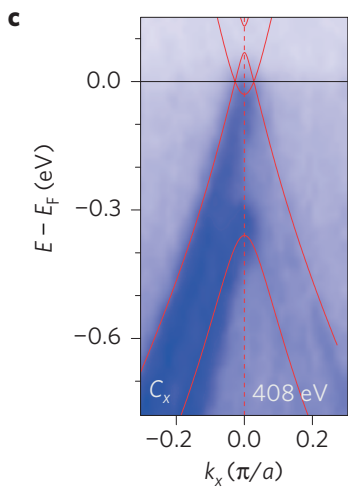

d

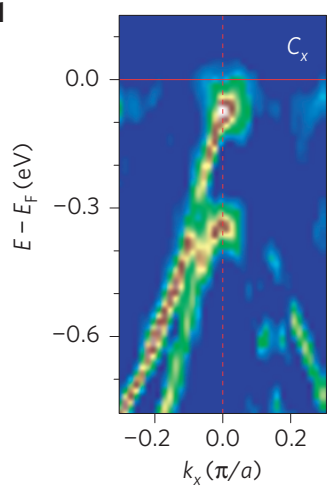

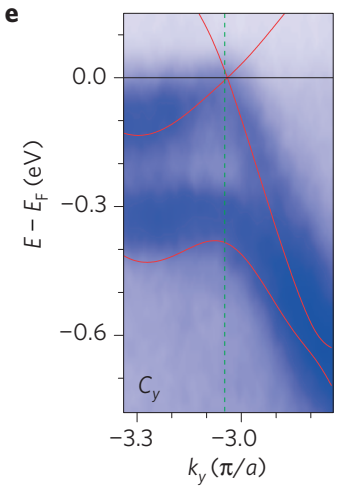

$\mathbf{f}$

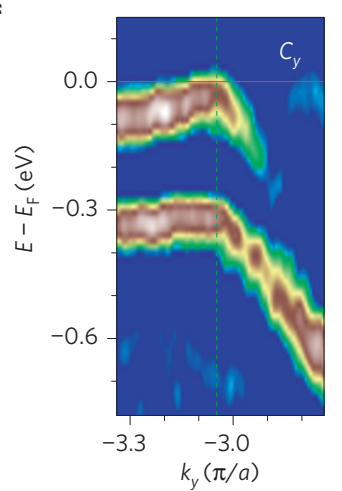

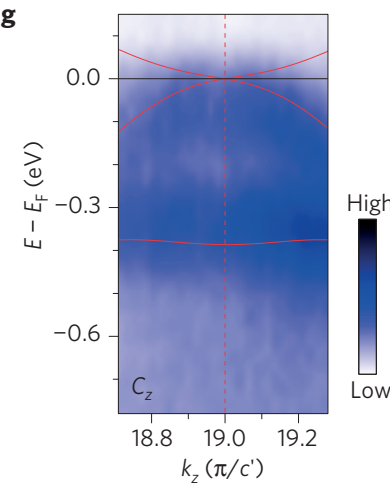

h

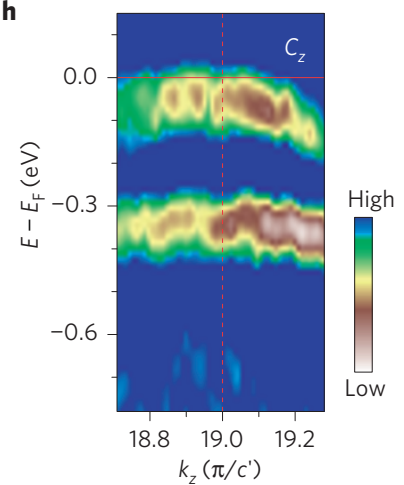

Figure 4 | Band dispersions near the Weyl nodes W2. a, Photoemission intensity plot at $E_{\mathrm{F}}$ in the $k_{\mathrm{y}}-k_{z}$ plane at $k_{x}=0$. The black curve indicates the

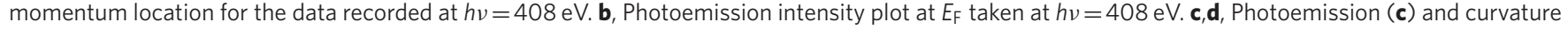

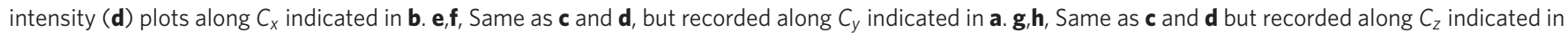
a. For comparison, the calculated bands along the $k_{x}, k_{y}$ and $k_{z}$ directions through the Weyl nodes W2 are plotted (red curves) on top of the experimental data in $\mathbf{c}, \mathbf{e}$ and $\mathbf{g}$, respectively. 

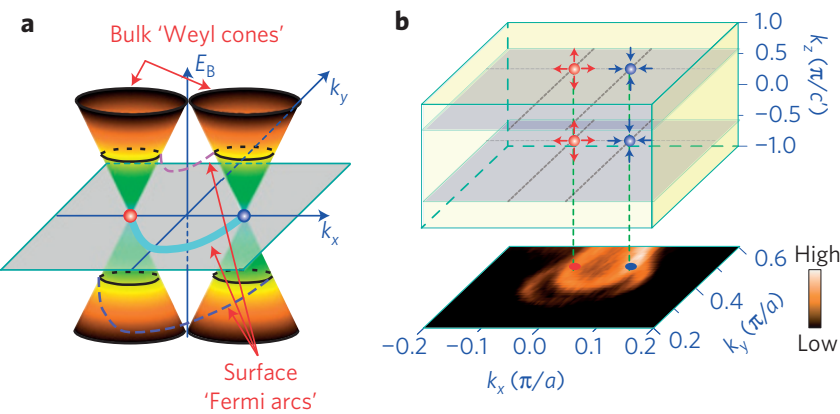

Figure 5 | Relationship between bulk Weyl nodes and surface Fermi arcs. a, Surface projection of a pair of bulk Weyl cones with opposite chirality connected by a surface Fermi arc. Blue, cyan and magenta curves are Fermi arcs at different binding energies. $\mathbf{b}$, Locations of experimentally determined Weyl nodes W1 in the 3D Brillouin zone and their projections onto the measured (001) surface Fermi arcs. The projections of the Weyl nodes are connected to the Fermi arcs, thus proving the existence of Weyl fermions in TaAs. The Weyl nodes are located at $( \pm 0.06,0.47,0.58)$ in units of $\left(\pi / a, \pi / a, \pi / c^{\prime}\right)$. Red and blue colours represent Weyl nodes with opposite chirality. The arrows indicate that the Weyl node is a monopole of Berry flux in the momentum space. The Fermi arcs are obtained at $h v=54 \mathrm{eV}$ using circularly polarized light.

and $k_{z}$ directions, with a much smaller slope along $k_{z}$, which is consistent with the band calculations. The excellent agreement between the experimental and calculated band dispersions along all three directions supports the existence of the Weyl nodes W2. From the measured dispersions, the Weyl nodes W2 are determined to be at $(\sim 0, \pm 1.05,0)$, which are very close to the calculated locations $( \pm 0.01, \pm 1.03,0)$ (ref. 10).

Finally, it is crucial to verify experimentally the relationship between the bulk Weyl nodes and the surface Fermi arcs. When a pair of bulk Weyl cones with opposite chirality are projected onto the surface, there must be a surface Fermi arc connecting them (Fig. $5 a)^{6}$. As shown in Fig. 5b, the projections of the Weyl nodes $\mathrm{W} 1$ onto the (001) surface BZ are exactly located on the loci of the Fermi arcs observed by our VUV ARPES (ref. 23). Therefore, our results have provided convincing experimental evidence that Weyl nodes in certain projected surfaces are the 'source' (or 'drain') for the surface Fermi arcs, which is a hallmark of a WSM.

Note added in proof: After acceptance of our paper, we became aware of a related manuscript (ref. 31) showing similar results to this work and those of refs 23,24

\section{Methods}

Methods and any associated references are available in the online version of the paper.

Received 7 April 2015; accepted 29 June 2015; published online 17 August 2015

\section{References}

1. Weyl, H. Elektron und gravitation. Z. Phys. 56, 330-352 (1929).

2. Balents, L. Weyl electrons kiss. Physics 4, 36 (2011).

3. Wang, Z. et al. Dirac semimetal and topological phase transitions in $\mathrm{A}_{3} \mathrm{Bi}$ (A = Na, K, Rb). Phys. Rev. B 85, 195320 (2012).

4. Wang, Z., Weng, H., Wu, Q., Dai, X. \& Fang, Z. Three-dimensional Dirac semimetal and quantum transport in $\mathrm{Cd}_{3} \mathrm{As}_{2}$. Phys. Rev. B 88, 125427 (2013).

5. Fang, Z. et al. The anomalous Hall effect and magnetic monopoles in momentum space. Science 302, 92-95 (2003).

6. Wan, X., Turner, A. M., Vishwanath, A. \& Savrasov, S. Y. Topological semimetal and Fermi-arc surface states in the electronic structure of pyrochlore iridates. Phys. Rev. B 83, 205101 (2011).

7. Xu, G., Weng, H., Wang, Z., Dai, X. \& Fang, Z. Chern semimetal and the quantized anomalous Hall effect in $\mathrm{HgCr}_{2} \mathrm{Se}_{4}$. Phys. Rev. Lett. 107, 186806 (2011).
8. Nielsen, H. B. \& Ninomiya, M. Absence of neutrinos on a lattice: (i). Proof by homotopy theory. Nucl. Phys. B 185, 20-40 (1981).

9. Nielsen, H. B. \& Ninomiya, M. Absence of neutrinos on a lattice: (ii). Intuitive topological proof. Nucl. Phys. B 193, 173-194 (1981).

10. Weng, H., Fang, C., Fang, Z., Bernevig, B. A. \& Dai, X. Weyl semimetal phase in noncentrosymmetric transition-metal monophosphides. Phys. Rev. X 5, 011029 (2015).

11. Huang, S. M. et al. An inversion breaking Weyl semimetal state in the TaAs material class. Nature Commun. 6, 7373 (2015).

12. Burkov, A. A. \& Balents, L. Weyl semimetal in a topological insulator multilayer. Phys. Rev. Lett. 107, 127205 (2011).

13. Halász, G. B. \& Balents, L. Time-reversal invariant realization of the Weyl semimetal phase. Phys. Rev. B 85, 035103 (2012).

14. Zyuzin, A. A., Wu, S. \& Burkov, A. A. Weyl semimetal with broken time reversal and inversion symmetries. Phys. Rev. B 85, 165110 (2012).

15. Lu, L., Fu, L., Joannopoulos, J. D. \& Soljačíc, M. Weyl points and line nodes in gyroid photonic crystals. Nature Photon. 7, 294-299 (2013).

16. Lu, L. et al. Experimental observation of Weyl points. Science http://dx.doi.org/10.1126/science.aaa9273 (2015).

17. Dubček, T. et al. Weyl points in three-dimensional optical lattices: Synthetic magnetic monopoles in momentum space. Phys. Rev. Lett. 114, 225301 (2015).

18. Hirayama, M., Okugawa, R., Ishibashi, S., Murakami, S. \& Miyake, T. Weyl node and spin texture in trigonal tellurium and selenium. Phys. Rev. Lett. 114, 206401 (2015)

19. Liu, J. \& Vanderbilt, D. Weyl semimetals from noncentrosymmetric topological insulators. Phys. Rev. B 90, 155316 (2014).

20. Bulmash, D., Liu, C.-X. \& Qi, X.-L. Prediction of a Weyl semimetal in $\mathrm{Hg}_{1-x-y} \mathrm{Cd}_{x} \mathrm{Mn}_{y}$ Te. Phys. Rev. B 89, 081106 (2014).

21. Singh, B. et al. Topological electronic structure and Weyl semimetal in the $\mathrm{TlBiSe}_{2}$ class of semiconductors. Phys. Rev. B 86, 115208 (2012).

22. Bzdušek, T., Rüegg, A. \& Sigrist, M. Weyl semimetal from spontaneous inversion symmetry breaking in pyrochlore oxides. Phys. Rev. B 91, 165105 (2015).

23. Lv, B. Q. et al. Experimental discovery of Weyl semimetal TaAs. Phys. Rev. X 5, 031013 (2015).

24. Xu, S. Y. et al. Discovery of a Weyl Fermion semimetal and topological Fermi arcs. Science http://dx.doi.org/10.1126/science.aaa9297 (2015).

25. Huang, X. C. et al. Observation of the chiral anomaly induced negative magneto-resistance in 3D Weyl semi-metal TaAs. Preprint at http://arXiv.org/abs/1503.01304 (2015).

26. Zhang, C. et al. Tantalum monoarsenide: An exotic compensated semimetal. Preprint at http://arXiv.org/abs/1502.00251 (2015).

27. Nielsen, H. B. \& Ninomiya, M. The Adler-Bell-Jackiw anomaly and Weyl fermions in a crystal. Phys. Lett. B 130, 389-396 (1983).

28. Son, D. T. \& Spivak, B. Z. Chiral anomaly and classical negative magnetoresistance of Weyl metals. Phys. Rev. B 88, 104412 (2013).

29. Hosur, P. \& Qi, X. Recent developments in transport phenomena in Weyl semimetals. C. R. Phys. 14, 857-870 (2013)

30. Strocov, V. N. Intrinsic accuracy in 3-dimensional photoemission band mapping. J. Electron Spectrosc. Relat. Phenom. 130, 65-78 (2003).

31. Yang, L. X. et al. Weyl semimetal phase in the non-centrosymmetric compound TaAs. Nature Phys. http://dx.doi.org/10.1038/nphys3425 (2015).

\section{Acknowledgements}

We acknowledge help in plotting figures from H. Miao and W. L. Zhang. This work was supported by the Ministry of Science and Technology of China (Nos 2013CB921700, 2015CB921300, 2011CBA00108 and 2011CBA001000), the National Natural Science Foundation of China (Nos 11474340, 11422428, 11274362 and 11234014), the Chinese Academy of Sciences (No. XDB07000000), the Sino-Swiss Science and Technology Cooperation (No. IZLCZ2138954), and the Swiss National Science Foundation (No. 200021-137783).

\section{Author contributions}

H.D., T.Q. and M.S. conceived the experiments. B.Q.L., N.X. and J.Z.M. performed ARPES measurements with the assistance of C.E.M., F.B., V.N.S. and J.M. H.M.W., Z.F and X.D. performed $a b$ initio calculations. N.X., B.Q.L., J.Z.M., T.Q. and H.D. analysed the experimental data. N.X., B.Q.L. and H.M.W. plotted the figures. T.Q., H.D., H.M.W., M.S. and P.R. wrote the manuscript. X.C.H., L.X.Z. and G.F.C. synthesized the single crystals.

\section{Additional information}

Reprints and permissions information is available online at www.nature.com/reprints. Correspondence and requests for materials should be addressed to T.Q., M.S. or H.D.

\section{Competing financial interests}

The authors declare no competing financial interests. 


\section{Methods}

Sample synthesis. Single crystals of TaAs were grown by chemical vapour transport. A previously reacted polycrystalline TaAs sample was filled in a quartz ampoule using $2 \mathrm{mg} \mathrm{cm}^{-3}$ of iodine as transporting agent. After evacuating and sealing, the ampoule was kept at the growth temperature for three weeks. Large polyhedral crystals with dimensions up to $1.5 \mathrm{~mm}$ were obtained in a temperature field of $\Delta T=1,150^{\circ} \mathrm{C}-1,000^{\circ} \mathrm{C}$. The as-grown crystals were characterized by $\mathrm{X}$-ray diffraction (XRD) using a PANalytical diffractometer with $\mathrm{Cu} \mathrm{K} \alpha$ radiation at room temperature. Single-crystal XRD was used to determine the crystal growth orientation. The average stoichiometry was determined by energy-dispersive X-ray (EDX) spectroscopy. No $\mathrm{I}_{2}$ doping was detected.

Angle-resolved photoemission spectroscopy. Soft X-ray ARPES measurements were performed at the Advanced Resonant Spectroscopies (ADRESS) beamline at the Swiss Light Source (SLS) with a SPECS analyser, and data were collected using circularly polarized light with an overall energy resolution of the order of $50-80 \mathrm{meV}$ at $T=10 \mathrm{~K}$. VUV ARPES measurements were performed at the Surface and Interface (SIS) beamline at the SLS with a Scienta R4000 analyser. Fresh surfaces for ARPES measurements were obtained by cleaving TaAs samples in situ in a vacuum better than $5 \times 10^{-11}$ torr.

Band structure calculations. First-principles calculations were performed using the OpenMX (ref. 32) software package. We have checked that the choices of pseudo-atomic orbital basis set with Ta9.0-s2p2d2f1 and As9.0-s2p2d1, the pseudo-potentials for Ta and As and the sampling of the BZ $(10 \times 10 \times 10 \mathrm{k}$-grid $)$ describe the electronic structure accurately. The exchange-correlation functional within the generalized gradient approximation parameterized by Perdew, Burke and Ernzerhof has been used ${ }^{33}$. The optimized lattice constants $a=b=3.4824 \AA, c=11.8038 \AA$ and atomic sites are in agreement with the experimental values.

\section{References}

32. http://www.openmx-square.org

33. Perdew, J. P., Burke, K. \& Ernzerhof, M. Generalized gradient approximation made simple. Phys. Rev. Lett. 77, 3865-3868 (1996). 